\title{
The prevention, detection and management of cancer treatment-induced cardiotoxicity: a meta-review
}

\author{
Aaron Conway ${ }^{1}$, Alexandra L McCarthy ${ }^{2}$, Petra Lawrence ${ }^{3}$ and Robyn A Clark ${ }^{4^{*}}$
}

\begin{abstract}
Background: The benefits associated with some cancer treatments do not come without risk. A serious side effect of some common cancer treatments is cardiotoxicity. Increased recognition of the public health implications of cancer treatment-induced cardiotoxicity has resulted in a proliferation of systematic reviews in this field to guide practice. Quality appraisal of these reviews is likely to limit the influence of biased conclusions from systematic reviews that have used poor methodology related to clinical decision-making. The aim of this meta-review is to appraise and synthesise evidence from only high quality systematic reviews focused on the prevention, detection or management of cancer treatment-induced cardiotoxicity.
\end{abstract}

Methods: Using Cochrane methodology, we searched databases, citations and hand-searched bibliographies. Two reviewers independently appraised reviews and extracted findings. A total of 18 high quality systematic reviews were subsequently analysed, $67 \%(n=12)$ of these comprised meta-analyses.

Results: One systematic review concluded that there is insufficient evidence regarding the utility of cardiac biomarkers for the detection of cardiotoxicity. The following strategies might reduce the risk of cardiotoxicity: 1) The concomitant administration of dexrazoxane with anthracylines; 2) The avoidance of anthracyclines where possible; 3) The continuous administration of anthracyclines (>6 h) rather than bolus dosing; and 4) The administration of anthracycline derivatives such as epirubicin or liposomal-encapsulated doxorubicin instead of doxorubicin. In terms of management, one review focused on medical interventions for treating anthracycline-induced cardiotoxicity during or after treatment of childhood cancer. Neither intervention (enalapril and phosphocreatine) was associated with statistically significant improvement in ejection fraction or mortality.

Conclusion: This review highlights the lack of high level evidence to guide clinical decision-making with respect to the detection and management of cancer treatment-associated cardiotoxicity. There is more evidence with respect to the prevention of this adverse effect of cancer treatment. This evidence, however, only applies to anthracycline-based chemotherapy in a predominantly adult population. There is no high-level evidence to guide clinical decision-making regarding the prevention, detection or management of radiation-induced cardiotoxicity.

Keywords: Heart failure, Chemotherapy, Cardiotoxicity, Cancer, Systematic review, Meta-review

\footnotetext{
* Correspondence: robyn.clark@flinders.edu.au

${ }^{4}$ School of Nursing and Midwifery, Flinders University, 5042 GPO Box 2100,

Sturt Road, Bedford Park, Adelaide 5001, South Australia

Full list of author information is available at the end of the article
} 


\section{Background}

Numerous factors, such as the introduction of screening programs to facilitate early detection [1, 2], improved diagnostic imaging, advances in therapy and the implementation of multidisciplinary cancer care [3], have contributed to improved cancer survival rates over recent decades [4, 5]. Advances in chemo- and radiotherapy have had the most impact on cancer survival [6]. The benefits associated with some cancer treatments, however, do not come without risk. A devastating side effect of some common cancer treatments is cardiotoxicityprincipally heart failure. The risk of cardiotoxicity varies according to the type and intensity of cancer treatment. Heart failure incidence rates associated with the commonly-prescribed chemotherapy agents include 0.14-48 \% for anthracyclines (estimated risk for doxorubicin dose $>400 \mathrm{mg} / \mathrm{m}$ [2] ranges from $0.14 \%$ to $5 \%$; for $550 \mathrm{mg} / \mathrm{m} 2$ it ranges from $7 \%$ to $26 \%$, and for $700 \mathrm{mg} / \mathrm{m}^{2}$ the estimated risk ranges from $18 \%$ to $48 \%$ ) [7]. For high dose cyclophosphamides the risk ranges from 7 to $28 \%$ for high-dose cyclophosphamides [8]. The risk is $1 \%$ for trastuzamab (while $5 \%$ of patients develop systolic dysfunction, only $1 \%$ develop symptomatic cardiomyopathy) [7, 9]; and 8 to $12.5 \%$ for tyrosine kinase inhibitors $[10,11]$. Cardiotoxicity, which can occur up to 20 years after treatment $[12,13]$ is likely to become even more prevalent as the cancer population ages and novel, so-called 'targeted' treatment regimens that cause damage to cardiac myocytes are more commonly employed. Concomitant chest irradiation in blood, breast and lung cancers is also implicated in cardiotoxicity [14].

Growing recognition of the longer-term public health implications of this problem, which is expected to increase as more people successfully complete acute cancer treatment, has resulted in a great deal of research in this field. Two key strategies are commonly utilised to support implementation of evidence into clinical practice; clinical practice guidelines and literature reviews (including both systematic and non-systematic review methodology). Guidelines for preventing, monitoring and treating cancer treatment-induced cardiotoxicity are available [8]. Non-systematic reviews have been published to support clinical practice and research related to cancer treatment-induced cardiotoxicity [15]. In addition, a number of systematic reviews have been published on this issue. However, critical appraisal and synthesis of systematic reviews and meta-analyses is needed in order to ensure that decision-making is informed by the best available accumulated evidence [16]. The 'meta-review' employs a unique review methodology in which the findings presented in individual systematic reviews and metaanalyses are appraised and synthesized. Methods similar to a traditional systematic review, such as comprehensive literature searches and quality assessment by two reviewers, are used. The difference between a traditional systematic review, which may or may not also incorporate meta-analysis, is that a meta-review only considers results reported in systematic reviews and meta-analyses, not results from individual studies. We conducted a metareview of the systematic reviews and meta-analyses that have addressed the important issue of cancer treatmentinduced cardiotoxicity. Our aim was to appraise and synthesise the systematic reviews that have focused on the prevention, early detection and management of cancer treatment-induced cardiotoxicity in order to aid policy and practice decision-making.

\section{Methods}

Cochrane methodology was used to appraise and synthesise systematic reviews in this field [6]. Our meta-review included a comprehensive literature search. The relevant reviews identified were then analysed by categorising and comparing the populations, interventions, comparisons and outcomes that were reported for each review. In addition, the quality of each review was appraised using a validated tool [16].

\section{Information sources and search strategy}

The following databases were searched: CINAHL; Cochrane Database of Systematic Reviews; Joanna Briggs Institute library of systematic reviews; EMBASE; Health source nursing/academic edition; and MEDLINE. The database searches were supplemented with manual searching of reference lists plus a forward citation search using Google Scholar. Only reviews published in peer-reviewed journals were included in this review [17]. Census dates from January 1996 and October 2013 (inclusive) were set for all literature searches. Only articles written in full-text English were included [18]. Potentially relevant publications were retrieved in full-text for review purposes. The search used Boolean operators to combine free text terms and/or $\mathrm{MeSH}$ terms including cardiotoxicity and systematic review. An example of the search terms used in one of the databases searched is presented in Additional File 1.

\section{Study selection}

Titles and abstracts were screened to eliminate irrelevant articles. Potentially eligible publications were retrieved and the full text version was reviewed in detail. Two reviewers independently selected studies for inclusion with a third independent reviewer was available for arbitration. Inclusion and exclusion criteria for this metareview are outlined in Table 1. 
Table 1 Inclusion and exclusion criteria for systematic reviews in this meta-review

Exclusion criteria - Systematic reviews focused on identifying the incidence of cardiotoxicity associated with particular cancer treatment regimens. - Poor quality (Literature search was not comprehensive, quality of included studies was not appraised, total AMSTAR score <7)

\section{Data extraction}

In addition to extracting data to describe the characteristics of each systematic review, such as the number of studies included, year of publication and the total number of participants, data about the populations, interventions, comparisons and outcomes were extracted. These data were extracted with a standardised form developed specifically for this study by two reviewers.

\section{Quality appraisal}

All potentially relevant reviews were appraised by two independent reviewers for their quality and risk of bias using the validated AMSTAR tool [16]. The 11 items of the AMSTAR were developed by building on empirical data collected with previously developed tools and with expert opinion. As such, the AMSTAR provided a valid, standardised method to assess the quality of methods used to search the literature and combine results, as well as the comprehensiveness that results of the reviews were reported [7]. Importantly, the AMSTAR criteria also provided a standardised method to determine the extent to which the scientific quality of the studies was assessed in the systematic reviews. The Cochrane Collaboration specify this as an important element to include in the preparation of a Cochrane overview of reviews [19]. Our definition of 'high-quality' was a review that addressed at least 7 of the 11 AMSTAR criteria. We deemed that setting a cut-off for the total score to indicate quality was appropriate, as psychometric testing of the AMSTAR tool revealed that, as each component score measures a different domain of quality, the summary score is meaningful [20]. Detailed results of appraisal of all relevant systematic reviews are presented in Additional File 2.

\section{Data synthesis}

Data extracted from the systematic reviews were categorised and presented in tables and forest plots. Summary findings are presented in a narrative synthesis.

\section{Results}

Overall, 31 publications from 352 citations were identified as potentially relevant. Of note, 11 relevant systematic reviews were judged to be of poor quality according to the AMSTAR criteria and were therefore excluded from this meta-review. Eighteen systematic reviews fulfilled the inclusion and exclusion criteria (Fig. 1).

\section{Systematic review characteristics}

The majority of reviews included randomized controlled trials [21-35], with only two reviews (11\%) also including prospective cohort designs [36, 37] (Table 2). The mean number of studies included in the reviews was 14.9 (range $=2-55$ ). The majority of the systematic reviews $(n-12 ; 67 \%)$ pooled results from individual studies for meta-analysis [21-26, 28, 30-33]. The reviews that did not use meta-analysis used a narrative approach to synthesise the findings $(n=6 ; 33 \%)[27,34-38]$. The systematic reviews were published from 2004 to 2013.

\section{Key findings from systematic reviews Detection of cancer treatment-induced cardiotoxicity}

Only one systematic review focused on interventions to detect cancer treatment-induced cardiotoxicity [36]. This systematic review identified one randomized controlled trial and six cohort studies that investigated the role of cardiac biomarkers, such as brain natriuretic peptide, in the early detection of cardiotoxicity in children who received anthracycline therapy [36]. The authors reported that the overall quality of the evidence was poor, due to a lack of randomized controlled trials and small sample sizes [36]. Based on these findings, the authors of the systematic review concluded that no clear recommendations for practice could be made regarding the use of cardiac biomarkers for the early detection of anthracycline-induced cardiotoxicity [36]. However, it is important to note that this review was published in 2007, with the literature search only current to January 2006. 


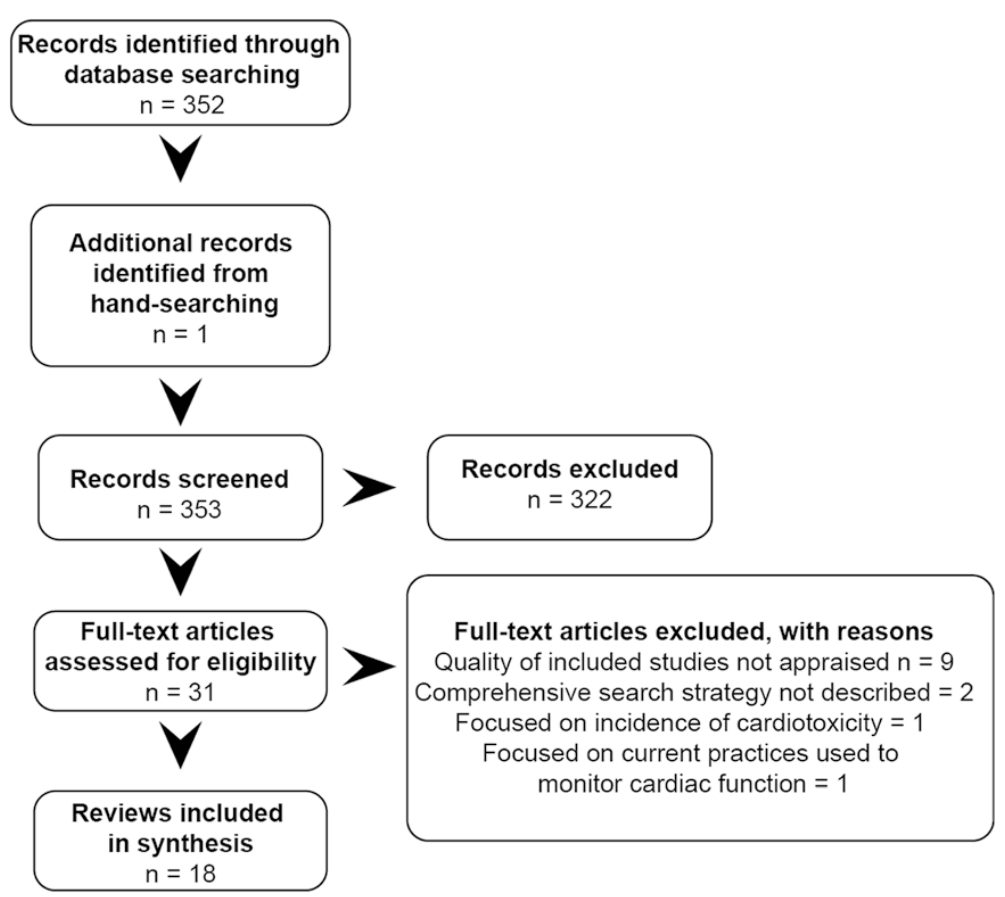

Fig. 1 Prisma flow chart - search results

\section{Prevention of cancer treatment-induced cardiotoxicity}

The majority ( $\mathrm{n}=16 ; 89 \%)$ of the systematic reviews investigated strategies to prevent cancer treatmentinduced cardiotoxicity [21-35, 37]. These reviews were further categorized into the following: Prevention of

1. Cardiotoxicity specifically associated with breast cancer treatment [21-26]

2. Cardiotoxicity specifically associated with prostate cancer treatment [27];

3. Anthracycline-induced cardiotoxicity in adult cancer patients [28, 30-33]

4. Cardiotoxicity through dietary supplementation [34]; and

5. Cancer treatment-induced cardiotoxicity in children [28, 35, 37].

Prevention-focused systematic reviews reported clinical cardiotoxicity, defined as the diagnosis of heart failure by a physician or a decline in left ventricular ejection fraction below $40 \%$, and sub-clinical cardiotoxicity. Definitions of sub-clinical cardiotoxicity varied considerably across reviews. For example, reviews used histological [30, 31], electrocardiographic [34] or echocardiographic [30-32] measurements to identify the presence of myocardial necrosis as a marker of subclinical cardiotoxicity.
The forest plot presented in Fig. 2 displays the results from meta-analyses that examined the effectiveness of different chemotherapy regimens or cardioprotective agents in the prevention of clinical cardiotoxicity. Differences between systematic reviews in their definition of what constituted sub-clinical cardiotoxicity precluded the formation of a similar figure for this outcome.

\section{Prevention of cardiotoxicity associated with breast cancer treatment}

Two of the breast cancer systematic reviews focused on taxane-based chemotherapy [21, 22]. In one pooled analysis of the results of 7 trials, there was no statistically significant difference in the rate of cardiotoxicity between adjuvant chemotherapy regimens with or without taxanes in women with early or operable breast cancer (OR 0.95; $95 \% \mathrm{CI}=0.67-1.36$ ) [21]. An earlier systematic review, which also examined the adverse effects of taxane-based adjuvant chemotherapy in women with early breast cancer, produced similar results [22]. Metaanalysis of 6 trials including 11,577 patients of adjuvant chemotherapy including a taxane revealed that the risk for development of cardiotoxicity was 11 per 1,000 (95\% $\mathrm{CI}=6-18$ ) [22]. In comparison, the risk for cardiotoxicity in women with early breast cancer who received adjuvant chemotherapy without a taxane was 12 per 1,000 [22]. The relative risk was $0.9(95 \% \mathrm{CI}=0.53-1.54)$ [22]. Of 
Table 2 Characteristics of included reviews

\begin{tabular}{|c|c|c|c|c|c|c|}
\hline Author (Year) & $\mathrm{PICO}$ & $\begin{array}{l}\text { Characteristics of } \\
\text { included studies }\end{array}$ & Intervention details & Summary of findings & $\begin{array}{l}\text { Meta- } \\
\text { analysis }\end{array}$ & $\begin{array}{l}\text { AMSTAR } \\
\text { score }\end{array}$ \\
\hline \multicolumn{7}{|c|}{ Detection } \\
\hline \multirow[t]{6}{*}{$\begin{array}{l}\text { Bryant et al. } \\
\text { (2007) [36] }\end{array}$} & $\begin{array}{l}\text { P: Children receiving } \\
\text { anthracyclines }\end{array}$ & $\begin{array}{l}\text { - One controlled trial } \\
\text { and } 6 \text { cohort studies }\end{array}$ & $\cdot c \operatorname{ctnT}$ & $\begin{array}{l}\text { - C-TnT can be used to assess cardioprotection } \\
\text { using dexrazoxane }\end{array}$ & $n$ & 7 \\
\hline & I: Cardiac markers & $\begin{array}{l}\text { - Published from } 1983 \\
\text { to } 2005\end{array}$ & - echocardiography & $\begin{array}{l}\text { - ANP and BNP are elevated in children who } \\
\text { received anthracyclines }\end{array}$ & & \\
\hline & C: Healthy control group & \multirow{4}{*}{$\begin{array}{l}\text { - Length of follow-up } \\
\text { in the studies was not } \\
\text { reported }\end{array}$} & - ANP, BNP & \multirow{4}{*}{$\begin{array}{l}\text { - NT-pro-BNP levels higher in children receiving } \\
\text { anthracyclines and had cardiac dysfunction } \\
\text { compared to those without }\end{array}$} & & \\
\hline & O: Cardiac damagePublish & & - Serum lipid peroxide & & & \\
\hline & & & - Serum carnitine & & & \\
\hline & & & - NT-pro-BNP & & & \\
\hline
\end{tabular}

\section{Prevention of anthracycline-induced cardiotoxicity}

\author{
Van Dalen \\ et al. (2010) [30] \\ P: Cancer patients
}

I: Anthracycline derivative

C: Another anthracycline with the same infusion duration and peak dose. Other chemotherapy and radiotherapy involving the

heart region must have been

the same as the intervention group.

0: Anthracycline-induced heart failure, subclinical cardiac dysfunction, abnormalities in dysfunction, abnormalities in
cardiac function, tumor response, patient survival, other toxicities, quality of life.

Van Dalen et al. P: Cancer patients who received (2009) [31]
I: Dosage schedule (different peak dose or infusion duration)

C: Same anthracycline derivative with the same dose. Other chemotherapy and radiotherapy involving the heart region must

\section{- 8 controlled trials}

- Published from 1984 to 2004

\section{- Median length of follow-up ranged from 21 to 41 \\ months}

- 11 controlled trials

• Published from 1989-2008

- Length of follow-up ranged from 7 days to median of 9 years.
- Doxorubicin vs epirubicin

Doxorubicin vs liposomalencapsulated doxorubicin

- Epirubicin vs liposomalencapsulated doxorubicin

No difference in rate of clinical heart failure between epirubicin and doxorubicin $(\mathrm{RR}=0.36 ; 95 \% \mathrm{Cl}=0.12-1.11)$

- Lower rate of clinical heart failure $(R R=0.20$ $95 \% \mathrm{Cl} 0.05$ to 0.75$)$ and subclinical heart failure (RR $=0.38,95 \% \mathrm{Cl} 0.24$ to 0.59 ) associated with liposomal-encapsulated doxorubicin compared with doxorubicin.

- No significant difference in the occurrence of clinical and subclinical heart failure between epirubicin and liposomal-encapsulated doxorubicin $(\mathrm{RR}=1.13,95 \% \mathrm{Cl} 0.46$ to $2.77, p=0.80)$
- Infusion duration

- Peak doses (maximal dos received in one week)
- In meta-analysis of 5 studies with 557 patients, a lower rate of clinical heart failure was observed with an infusion duration of $6 \mathrm{~h}$ or longer as compared to a shorter infusion duration $(\mathrm{RR}=0.27 ; 95 \% \mathrm{Cl}=0.09$ to 0.81$)$

- No significant difference in the occurrence of heart failure for different peak doses of anthracyline chemotherapy 
Table 2 Characteristics of included reviews (Continued)

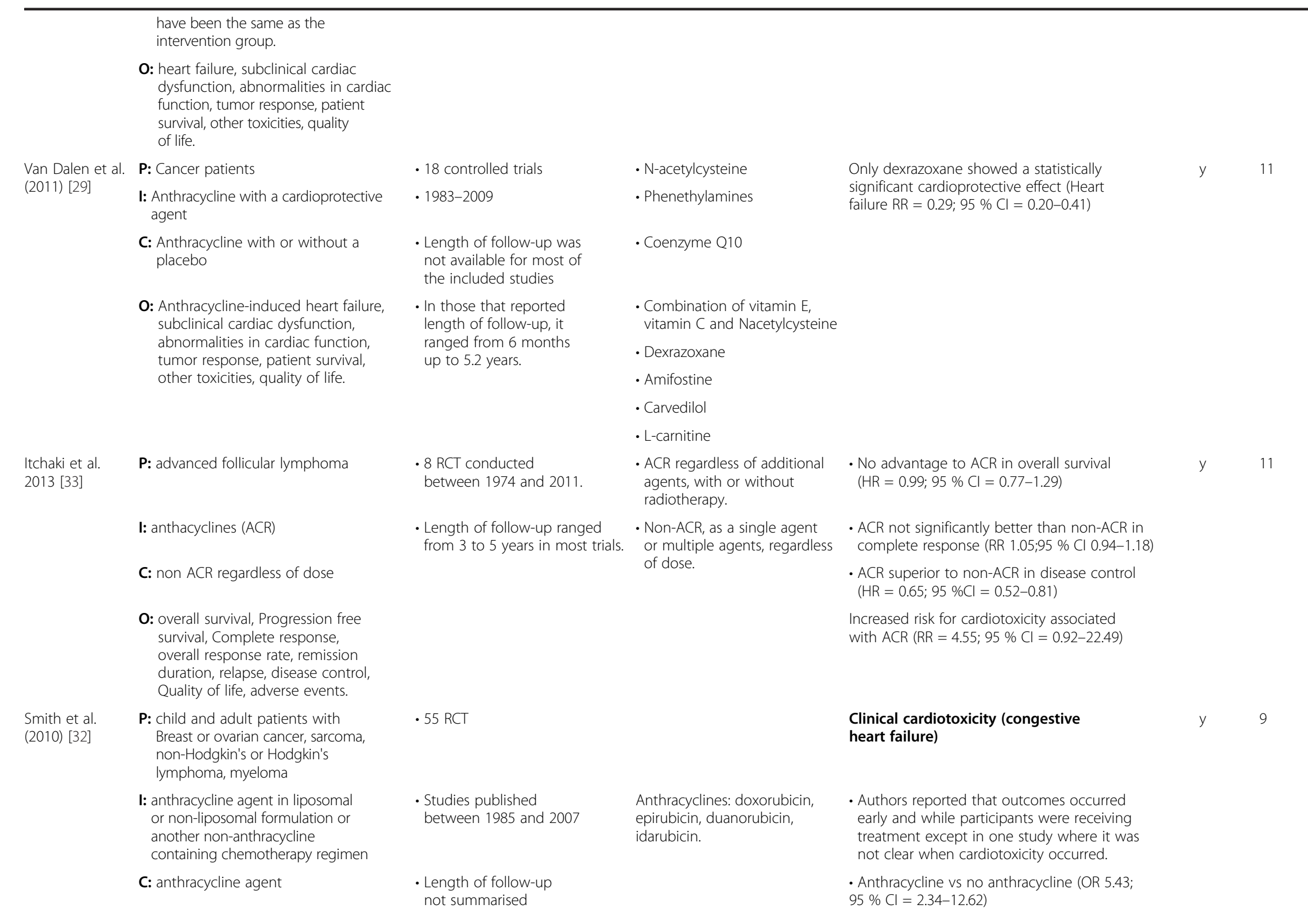

: Anthracycline-induced heart failure, cardiac dysfunction, umor response, patient survival, vitamin C and Nacetylcysteine

- Dexrazoxane

Amifostine

- Carvedilol

Itchaki et al. P: advanced follicular lymphoma
2013 [33]

8 RCT conducted agents, with or without adiotherapy.

政

Length of follow-up ranged

• ACR not significantly better than non-ACR in or multiple agents, regardless complete response (RR 1.05:95\% Cl 0.94-1.18)

ACR superior to non-ACR in disease control $(\mathrm{HR}=0.65 ; 95 \% \mathrm{Cl}=0.52-0.81)$

ncreased risk for cardiotoxicity associated overall response rate, remission Breast or ovarian cancer, sarcoma, non-Hodgkin's or Hodgkin's

: anthracycline agent in liposomal or non-liposomal formulation or another non-anthracycline

c: anthracycline agent 
Table 2 Characteristics of included reviews (Continued)

O: Clinical cardiotoxicity (diagnosis of chronic heart failure)

Subclinical cardiotoxicity (Reduction in left ventricular ejection fraction

or abnormality in cardiac function

determined using a diagnostic test)

\section{Dietary supplementation}

Roffe et a

Prevention of cardiotoxicity associated with

\section{prostate cancer treatment}

\section{Shelley et al \\ P: Hormone-refractory prostate (2008) [27]}

P: Cancer patients

I: Coenzyme Q10

C: Any comparison

O: All outcomes considered

- 6 controlled trials

(1 placebo-controlled double-blinded study, 5 open label)

- Published between

1982 and 1996

- Length of follow-up was not reported

- 47 RCT published between 1977 and 2005

- Length of follow up was not reported

C: Any comparison

O: Overall survival, Disease-specific survival, PSA response, time to progression, pain response, toxicity, quality of life.

- Bolus versus continuous infusion ( $\mathrm{OR}=4.13$;

$95 \% \mathrm{Cl}=1.75-9.72$

- Liposomal doxorubicin vs doxorubicin

$(\mathrm{OR}=0.18 ; 95 \% \mathrm{Cl}=0.08-0.38)$

- Epirubicin vs doxorubicin $\mathrm{OR}=0.39$

$(95 \% \mathrm{Cl}=0.2-0.78)$

- Anthracycline vs mitoxantrone $\mathrm{OR}=2.88$ $(95 \% \mathrm{Cl}=1.29-6.44)$

- Dexrazoxane vs no dexrazoxane $\mathrm{OR}=0.21$ (95\% Cl = 0.13-0.33)

- Anthracycline was associated with increased risk of sub-clinical cardiotoxicity $(\mathrm{OR}=6.25$; $95 \% \mathrm{Cl}=2.58-15.13)$

- Rate of cardiac deaths in 4 studies was significantly higher in the anthracycline groups $(\mathrm{OR}=4.94$. $95 \% \mathrm{Cl}=1.23-19.87, p=0.025$ )

Dose ranged from $30 \mathrm{mg}$ per day to $240 \mathrm{mg}$ per day

- Significant differences between groups

observed in various ECG measures.

- Effect on heart failure or subclinical cardiac dysfunction was not reported in the trials

Drug categories included:

- estramustine

$\cdot 5$-fluorouraci

- cyclophosphamide

- doxorubicin

- mitoxantrone

- docetaxel
Severe cardiovascular toxicity was more common with Estramustine versus Best Supportive Care or Hormones.

- Similar rates of cardiotoxicity with estramustine alone and medroxyprogesterone acetate plus epirubicin.

- Cardiotoxicity was less common with epirubicin (11\%) than doxorubicin (48\%).

- Doxorubicin combined with diethlystilbestrol was more cardiotoxic than doxorubicin (7 \% vs $1 \%$ ). 
Table 2 Characteristics of included reviews (Continued)

\section{Prevention in children}

Bryant et al. P: Children receiving anthracyclines (2007) [35]

I: Any cardioprotection intervention

C: Any comparison

O: Mortality, heart failure, arrhythmia, measures of cardiac function and cost-effectiveness

Sieswerda et al. P: children with cancer $2011[37]$

I: liposomal anthracyclines

C: Any comparison

O: cardiotoxicity, tumour response, adverse events

Van dalen et al. P: children with cancer 2012 [28]

I: anthracyclines

C: non anthracycline

\section{O: survival}

Tumour response cardiotoxicity

\section{Prevention of cardiotoxicity associated with}

\section{breast cancer treatment}

Valachis et al. P: Breast cancer

(2013) [24]
- 4 controlled trials published between 1994 and 2004

- Length of follow-up months

- Dexrazoxane

15 observational studies published between 1998 and 2007

- (9 prospective cohort studies, 2 retrospective cohort studies, three cas reports, one unclear design

- Duration of follow up was reported in 10 studies (ranged from to 58 months)

8 RCT published from 1975 to 2009

Length of follow-up was not mentioned in the majority of trials
- Different liposoma anthracyclines looked at Liposomal daunorubicin pegylated liposomal doxorubicin, liposomal doxorubicin

1153 treatment, 1121 control.

Ulmulative duanorubicin treatment protocol 90-350 $\mathrm{mg} / \mathrm{m} 2$.

Peak dose of anthracycline in one week = 25-90 mg/m2. doxorubicin treatment protocol was $300-420 \mathrm{mg} / \mathrm{m} 2$.

Peak dose doxorubicin in 1 week 25-60 mg/m2
- No cost-effectiveness data were identified in the systematic review

- There were conflicting results in trials of rapid or continuous infusion of anthracycline chemotherapy

- Coenzyme Q10 was examined in one small trial $(n=20)$

- Mean reduction in percentage left ventricular fraction shortening was lower in the group that received coenzyme Q10.

- Dexrazoxane was examined in a trial with 105 participants.

- Fewer patients who received dexrazoxane had elevations in troponin ( $21 \%$ vs $50 \%$; $p<0.001$ )

No evidence from controlled trials was identified.

Impossible to know whether there are differences in outcomes

- Rate of cardiac death was similar between treatment groups in meta-analysis of two trials $(\mathrm{RR}=0.41 ; 95 \% \mathrm{Cl}=0.04-3.89)$

- No significant difference in HF between treatment groups in one trial $(\mathrm{RR}=0.33 ; 95 \% \mathrm{Cl}=0.01-8.02)$ 
Table 2 Characteristics of included reviews (Continued)

\begin{tabular}{|c|c|c|c|c|c|c|}
\hline & & $\begin{array}{l}\text { - } 6 \text { controlled trials that } \\
\text { were all published in } \\
2012 \text {. }\end{array}$ & $\begin{array}{l}\text { Anti-HER2 monotherapy } \\
\text { (lapatinib or trastuzumab or } \\
\text { pertuzumab) }\end{array}$ & $\begin{array}{l}\text { monotherapy was } 0.58 \text { (95 \% Cl: } 0.26-1.27 \text {, } \\
p \text {-value }=0.17)\end{array}$ & & \\
\hline & I: anti-HER2 monotherapy & \multirow[t]{3}{*}{$\begin{array}{l}\text { - Length of follow-up was } \\
\text { not reported. }\end{array}$} & & $\begin{array}{l}\text { - Pooled OR of LVEF decline with dual anti-HER2 } \\
\text { therapy versus anti-HER2 monotherapy was } 0.88 \\
(95 \% \mathrm{Cl}: 0.53-1.48, p \text {-value }=0.64)\end{array}$ & & \\
\hline & C: anti-HER2 combination therapy & & & \multirow{2}{*}{$\begin{array}{l}\text { - Comparable cardiac toxicity between these two } \\
\text { therapies }\end{array}$} & & \\
\hline & $\begin{array}{l}\text { O: LVEF decline less than } 50 \% \text { or more } \\
\text { than } 10 \% \text { from baseline, National } \\
\text { Cancer Institute Common Toxicity } \\
\text { Criteria Chronic heart failure grade } \\
3 \text { or more. }\end{array}$ & & & & & \\
\hline \multirow[t]{5}{*}{$\begin{array}{l}\text { Viani et al. } \\
2007\end{array}$} & P: HER-2-positive early breast cancer & $\begin{array}{l}\cdot 5 \text { RCT published in } 2005 \\
\text { and } 2006\end{array}$ & $\begin{array}{l}\text { Doxorubicin and } \\
\text { cyclophosphamide } \\
(\mathrm{AC})+\text { paclitaxel }(\mathrm{P})\end{array}$ & $\begin{array}{l}\text { - Meta-analysis of } 5 \text { trials of adjuvant trastuzumab } \\
\text { revealed a significant reduction in mortality } \\
(p<0.00001) \text {, recurrence }(p<0.00001) \text {, metastases } \\
(p<0.00001) \text { and second tumours }(p=0.007) \\
\text { compared with no trastuzumab }\end{array}$ & $\mathrm{y}$ & 10 \\
\hline & I: adjuvant trastuzumab & \multirow[t]{4}{*}{$\begin{array}{l}\text { - Length of follow-up } \\
\text { ranged from } 9 \text { to } 60 \text { months } \\
\text { after randomisation }\end{array}$} & $\begin{array}{l}\text { Docetaxel or vinorelbine + } \\
\text { fluorouracil, epirubicin and } \\
\text { cyclophosphanide. }\end{array}$ & $\begin{array}{l}\text { - Increased cardiotoxicity including symptomatic } \\
\text { cardiac dysfunction and asymptomatic decrease } \\
\text { in LVEF with trastuzumab compared to no } \\
\text { trastuzumab }\end{array}$ & & \\
\hline & C: any comparison & & Doxo, cyclo + trastuz. & \multirow{3}{*}{$\begin{array}{l}\text { - The likelihood of cardiac toxicity was } 2.45 \text { times } \\
\text { higher for trastuzumab compared with no } \\
\text { trastuzumab (statistically significant heterogeneity) }\end{array}$} & & \\
\hline & $\begin{array}{l}\text { O: mortality, recurrance, metastases, } \\
\text { second tumour no breast cancer rate }\end{array}$ & & $\begin{array}{l}\text { Docetaxel, carboplatin }+ \\
\text { trastuz. }\end{array}$ & & & \\
\hline & Cardiac toxicity and brain metastases & & AC + docetaxel. & & & \\
\hline \multirow[t]{5}{*}{$\begin{array}{l}\text { Qin et al. } \\
2011[21]\end{array}$} & P: node negative breast cancer & $\begin{array}{l}\text { - } 19 \text { RCT published from } \\
2003 \text { to } 2010\end{array}$ & $\begin{array}{l}\text { Taxane treatment vs non } \\
\text { taxane treatment }\end{array}$ & $\begin{array}{l}\text { - Disease free survival: taxane treatment HR 0.82, } \\
95 \% \mathrm{Cl} 0.76-0.88\end{array}$ & y & 10 \\
\hline & I: adjuvant taxane & \multirow{4}{*}{$\begin{array}{l}\text { - Median length of follow- } \\
\text { up ranged from } 35 \text { to } \\
102 \text { months }\end{array}$} & & $\begin{array}{l}\text { - Overall Survival: HR } 0.85,95 \% \mathrm{Cl} 0.78-0.92 \\
\text { favoured taxane }\end{array}$ & & \\
\hline & C: chemo without taxane & & & $\begin{array}{l}\text { • increased toxicity for neutropenia }(\mathrm{OR}=2.28 \\
95 \% \mathrm{Cl} 1.25-4.16), \text { fatigue }(\mathrm{OR}=2.10,95 \% \mathrm{Cl} \\
1.37-3.22) \text {, diarrhea }(\mathrm{OR}=2.16,95 \% \mathrm{Cl} 1.32-3.53) \text {, } \\
\text { stomatitis (OR 1.68, } 95 \% \mathrm{Cl} 1.04-2.71) \text {, oedema } \\
\text { (OR 6.61, } 95 \% \mathrm{Cl} 2.14-20.49)\end{array}$ & & \\
\hline & \multirow[t]{2}{*}{$\begin{array}{l}\text { O: disease free survival, overall } \\
\text { survival, drug related toxicityof } \\
\text { taxane }\end{array}$} & & & $\begin{array}{l}\text { - In pooled analysis of results from } 7 \text { trials, there } \\
\text { was no statistically significant difference in the } \\
\text { rate of cardiotoxicty between chemotherapy } \\
\text { regimens with or without taxanes (OR 0.95; } \\
95 \% \mathrm{Cl}=0.67-1.36 \text { ) }\end{array}$ & & \\
\hline & & & & $\begin{array}{l}\text { - taxane treatment showed significant reduction } \\
\text { in death and recurrence }\end{array}$ & & \\
\hline $\begin{array}{l}\text { Lord et al. } \\
2008 \text { [26] }\end{array}$ & P: metastatic breast cancer & $\begin{array}{l}\text { - } 34 \text { RCT published } \\
\text { between } 1974 \text { and } 2004\end{array}$ & $\begin{array}{l}\text { - Comparison between } \\
\text { anthracyclines and non- }\end{array}$ & $\begin{array}{l}\text { - } 23 \text { trials with } 4777 \text { patients that compared } \\
\text { anthracycline with non-antitumour antibiotic } \\
\text { regimens reported on cardiotoxicity. }\end{array}$ & $\mathrm{y}$ & 10 \\
\hline
\end{tabular}

revealed a significant reduction in mortality

$(p<0.00001)$, recurrence $(p<0.00001)$, metastases

$(p<0.00001)$ and second tumours $(p=0.007)$

- Increased cardiotoxicity including symptomatic cardiac dysfunction and asymptomatic decreas in LVEF with trastuzumab compared to no trastuzumab - higher for trastuzumab compared with no

o: mortality, recurrance, metastases, Cardiac toxicity and brain metastases

I: adjuvant taxane

RCT published from

Median length of followup ranged from 35 to

C: chemo without taxane between 1974 and 2004 regimens reported on cardiotoxicity. 
Table 2 Characteristics of included reviews (Continued)

I: anti-tumour antibiotics

C: chemo regimens without anti tumour antibiotics

O: overall survival, time to progression, response, quality of life, toxicity

\section{Ferguson et al. P: breast cancer} $2007[22]$

I: chemotherapy with taxane

C: chemotherapy without taxane

O: overall survival, disease free survival, toxicity, quality of life, cost effectiveness

Duarte et al. 2012 [25]

\section{P: breast cancer}

I: conventional chemotherapy

C: aggressive adjuvant chemo

O: overall survival, disease free survival, incidence of Common Toxicity Criteria Scale grades $3,4,5$

\section{Managemen}

Sieswerda et al. P: children with cancer

I: anthracycline induced cardiotoxicity medical interventions

C: placebo, other medical interventions, no treatment

O: overall survival, mortality due to HF, development of HF, adverse events and tolerability
- Length of follow-up was not reported in most

- Estimated length of

follow-up from survival

curves ranged from 2 to

102 months.

- 12 RCT published from 2002 to 2006

Length of follow-up was 43 to 69 months.

\section{- 4 RCT published between}

2003 and 2009

- Length of follow-up

ranged from 23

125 months

\section{RCT published in 2004} and 2008

Enalapril Vs placebo

- Phosphecreatine vs control treatment (vitamin $C$

adenosine tri-phosphate

vitamin $\mathrm{E}$, oral

co-enzyme Q10)

Combinations Taxane and anthracycline; anthracycline; combined neo-adjuvant and adjuvant chemo; adjuvant vs non-adjuvant therapy; stimulation factor; adjuvan tamoxifan prescribed for 5 years
- Patients who received anthracyclines were more

likely to develop cardiotoxicity $\mathrm{OR}=5.17$

$95 \% \mathrm{Cl}=3.16-8.48)$

- Overall survival was reported in 23 studies of anthracyclines. No statistically significant

difference in overall survival was observed

between the regimens ( $\mathrm{HR} 0.97,95 \% \mathrm{Cl} 0.91-1.04$ )

- The rate of cardiotoxicty was not reported in

the mitoxantrone comparison.

- No difference in the risk of developing cardiotoxicity between taxane containing and non-taxane containing regimens (OR 0.90, $95 \% \mathrm{Cl} 0.53$ to 1.55$)$ in meta-analysis of 6 studies involving 11557 patients.

Disease free survival: dose dense therapy significant improvement $(\mathrm{HR}=0.83 .95 \%$ $\mathrm{Cl}=0.73-0.95)$

- Dose dense chemotherapy not capable of improving overall survival ( $\mathrm{HR}=0.86$ : $95 \%$ Cl 0.73-1.01)

-Women who received a dose-dense chemotherapy regimen were not more likely to develop cardiotoxicity $(\mathrm{OR}=0.5$;

$95 \% \mathrm{Cl}=0.05-5.54)$

\section{. 203 patients in total}

- Median follow-up was 2.8 years

- One intervention participant developed clinically significant decline in cardiac performance compared with 6 control participants (RR $=0.16,95 \% \mathrm{Cl} 0.02-1.29$ ).

- Higher occurrence of dizziness or hypotension (RR 7.17, $95 \% \mathrm{Cl} 1.71$ to 30.17 ) associated with enalapril 
Legend: CTnT Cardiac Troponin T, ANP Atrial Natriuretic Peptide, NT-BNP N-terminal Brain Natriuretic Peptide, ACR, anthacyclines, LVEF Left ventricular ejection fraction, HF Heart failure, $95 \%$ CI $95 \%$ Confidence Interval, $R R$ Relative risk, $O R$, Odds ratio, $H R$ Hazard ratio, $R C T$ Randomised controlled trial.

\section{Phosphocreatine trial}

- Length of follow-up estimated to be 15 days

- No deaths in both groups

- No adverse events reported

- no definitive conclusions can be drawn

due to small sample size 


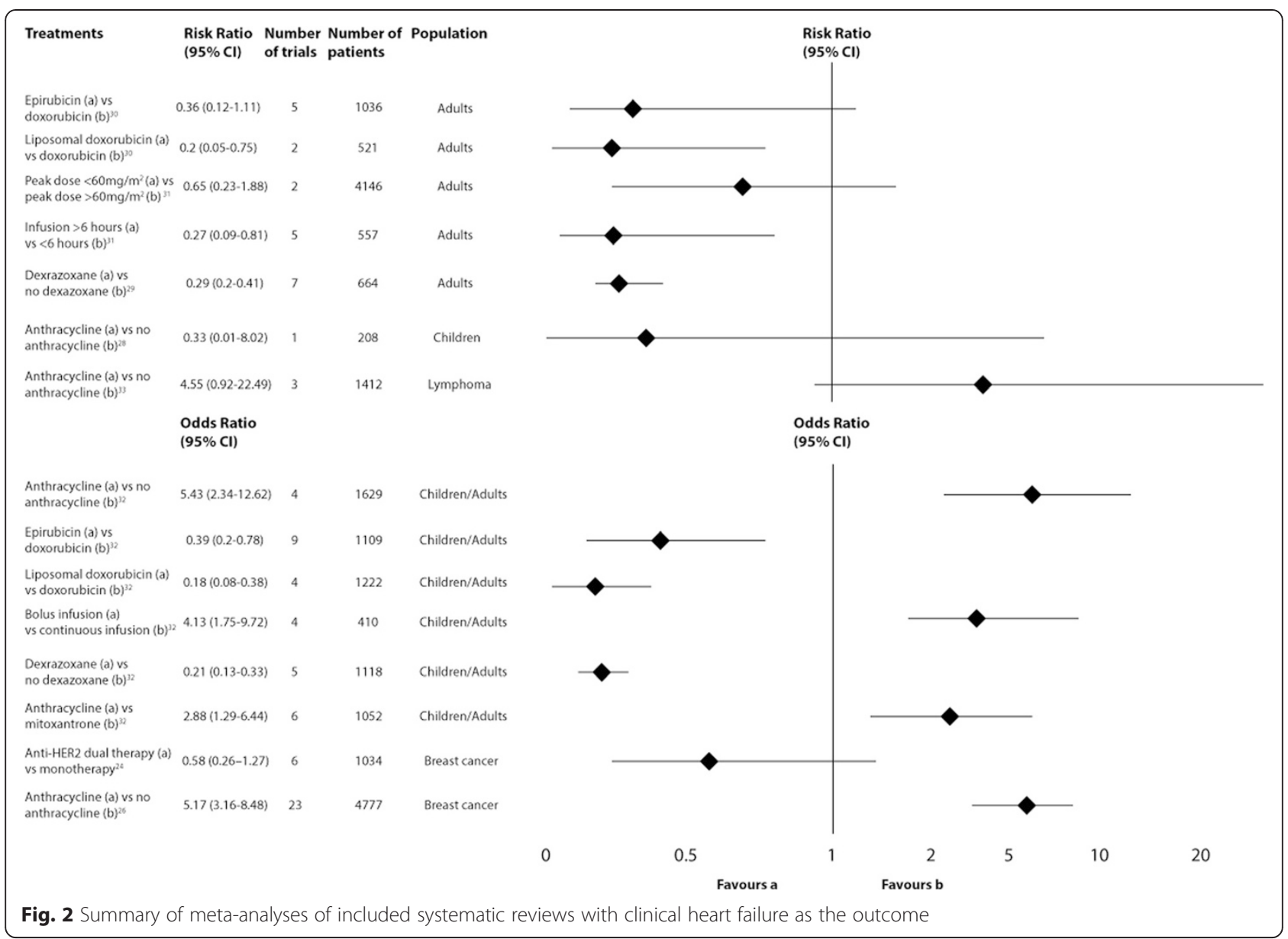

note, the chemotherapy regimens of control and intervention arms of the studies included in the meta-analyses contained anthracyclines [21, 22]. As there were no differences in the rate of cardiotoxicity between participants who did and did not receive taxanes, it would appear that the rates of cardiotoxicity were likely due to the use of anthracyclines.

A specific focus of two further systematic reviews that examined the prevention of cardiotoxicity associated with breast cancer treatment investigated the impact of anti-human epidermal growth factor receptor 2 (HER-2) therapy [23, 24]. One systematic review aimed to determine whether there was an increased risk of cardiotoxicity in breast cancer patients treated with dual HER-2 blockade (pertuzumab plus trastuzumab, or trastuzumab plus lapatanib) compared to monotherapy (lapatanib or trastuzumab or pertuzumab) [24]. No statistically significant difference in the likelihood of developing clinical heart failure or of decline in left ventricular ejection fraction was identified [24]. The authors concluded that the evidence supported the use of dual therapy in this population with no adverse cardiac effects. The second breast cancer systematic review focused on the anti-HER-2 medication trastuzumab [23]. Meta-analysis indicated that the odds of developing cardiotoxicity were 2.45 times higher $(95 \% \mathrm{CI}=1.89-3.16)$ in subjects prescribed trastuzumab [23]. However, overall mortality, recurrence and mortality rates were decreased in subjects who received trastuzamab despite their higher odds of developing post-treatment cardiac symptoms. [23] Close monitoring of cardiac function was recommended, based on these findings.

A systematic review of randomized controlled trials of dose-dense anthracycline-based chemotherapy in early breast cancer, comprising a meta-analysis that combined $n=1,310$ patients, revealed that women who received a dose-dense chemotherapy regimen were not more likely to develop cardiotoxicity $(\mathrm{OR}=0.5 ; 95 \%$ $\mathrm{CI}=0.05-5.54)$ [25]. Trials of dose-dense chemotherapy were defined in this review as the same type and total amount of the drug administered over a shorter interval of time [25]. A further systematic review focused 
on anthracycline regimens for metastatic breast cancer [26]. The authors recommended careful consideration regarding the use of anthracyclines in this population due to the increased likelihood of developing cardiotoxicity (OR $=5.17 ; 95 \% \mathrm{CI}=3.16-8.48)[26]$.

\section{Prevention of cardiotoxicity associated with prostate cancer treatment}

One systematic review addressed the issue of cardiotoxicity associated with treatment for hormone-refractory prostate cancer [27]. While 47 trials met the inclusion criteria for this review, the chemotherapy regimens investigated were too dissimilar to conduct meta-analysis [27]. No specific recommendations for the prevention of cardiotoxicity in this population were noted by the authors of this review.

\section{Prevention of anthracycline-induced cardiotoxicity in adult cancer patients}

In a systematic review of cardioprotective agents used during anthracycline therapy, the authors concluded that of the eight different agents for which there were randomized controlled trials, only dexrazoxane could be recommended for clinical practice [29]. Conflicting recommendations were provided in another systematic review by Smith et al. [32], which concluded that the evidence was not sufficiently robust to support the routine use of any particular cardioprotective agent, nor a liposomal formulation or alternative anthracycline treatment regimen. In regard to the use of anthracycline derivatives, a 2010 systematic review by van Dalen et al. came to a similar conclusion, suggesting that further research is needed to provide recommendations regarding the use of these alternative chemotherapy regimens [30].

Anthracycline administration considerations were examined for their potential association with cardiotoxicity in a second systematic review by van Dalen [31]. This review identified that continuous anthracycline infusion $(>6 \mathrm{~h})$ rather than bolus injection reduced the risk of cardiotoxicity [31]. No differences were observed in the rate of cardiotoxicity as a result of different doxorubicin peak doses [31].

A recently published review (2013) by Itchaki focused on anthracycline use in people receiving treatment for advanced follicular lymphoma [33]. Due to the increased risk ratio for cardiotoxicity $(4.55 ; 95 \% \mathrm{CI}=0.92-22.49)$ associated with anthracycline treatment in this population, the authors concluded that evidence of the benefit of anthracyclines in this population is limited [33].

\section{Dietary supplementation}

One systematic review appraised randomized and nonrandomized studies that reported the use of coenzyme Q10 (CoQ10) to reduce the adverse effects of cancer treatment [34]. Only three randomized controlled trials, which included a total of 140 patients, investigated the effects of CoQ10 on cardiotoxicity [34]. These trials were not subjected to meta-analysis. The authors of the systematic review concluded that CoQ10 could provide some protection against cardiotoxicity during cancer treatment based on the fact that significant differences in electrocardiographic measurements were identified between control and CoQ10 groups [34]. However, using CoQ10 in clinical practice was not recommended, due to insufficient data [34].

\section{Prevention of cancer treatment-induced cardiotoxicity in children}

All of the systematic reviews that focused on the prevention of cardiotoxicity in children addressed this issue as it related to anthracycline-based chemotherapy. Sieswerda et al. [37] concluded that randomized controlled trials are needed to increase understanding of the benefits and risks of liposomal anthracyclines in children, as the evidence to date solely consists of observational studies [37]. In a further systematic review, metaanalysis of two randomized controlled trials revealed no statistically significant difference in the risk of cardiac death $(\mathrm{RR}=0.4 ; 95 \% \mathrm{CI}=0.04-3.89)$ or heart failure $(\mathrm{RR}$ $=0.33 ; 95 \% \mathrm{CI}=0.01-8.02)$ in children who received anthracyclines [28]. However, the total number of participants in the randomized controlled trials was small (n $=410$ ) [28]. As such, no firm conclusions regarding the implications for clinical practice were drawn from this analysis. A further systematic review focused on cardioprotection in children who received anthracyclines [35]. Based on the fact that only four randomized controlled trials with methodological limitations met the inclusion criteria, the authors concluded that there was limited evidence to guide cardioprotective therapies in this population and definitive recommendations for practice could not be made [35].

\section{Management of cancer treatment-induced cardiotoxicity}

Only one systematic review focused on interventions to treat cancer treatment-induced cardiotoxicity [38]. This review focused on the treatment of anthracyclineinduced cardiotoxicity in children. Only two randomized controlled trials, which enrolled a total of 203 patients, were included in this review. The two interventions tested were enalapril and phosphocreatine. While the participants who received enalapril were less likely to experience decline in cardiac function, the difference between groups was not statistically significant $(p<0.5)$. Moreover, participants who received enalapril were more likely to experience hypotension, dizziness and fatigue. Therefore, the authors concluded that the benefits of this therapy be weighed against the greater risk of side 
effects in children with asymptomatic cardiotoxicity [38]. Conclusions regarding the use of phosphocreatine could not be made due to the high risk of bias. The authors of the review concluded that further high quality randomized controlled trials are required in this field [38].

\section{Discussion}

This aim of this meta-review was to appraise and synthesise the systematic reviews that have focused on the prevention, early detection and management of cancer treatment-induced cardiotoxicity in order to aid policy and practice decision-making. Based on the 18 systematic reviews included in this meta-review that were deemed to be high quality according to the AMSTAR criteria, the following conclusions can be drawn. First, there is insufficient evidence to draw firm conclusions regarding the utility of cardiac biomarkers for the detection of cancer treatment-induced cardiotoxicity. Based on conclusions drawn from systematic reviews focused on prevention, the following strategies could reduce the risk of cardiotoxicity: 1) The concomitant administration of dexrazoxane with anthracylines; 2) The administration of continuous anthracyclines, preferably for longer than $6 \mathrm{~h}$, rather than bolus dosing; and 3) The administration of anthracycline derivatives such as epirubicin or liposomal-encapsulated doxorubicin instead of doxorubicin. In this context, it should be noted that while dexrazoxane is listed in the relevant pharmaceutical benefits scheme in some countries for this indication (e.g. it is listed as such by the FDA in the USA), in others such as Australia it is not. Hence its routine use would be problematic in some countries, as it would be hard to procure and expensive for patients who already incur considerable treatment overheads. In addition, in many high volume chemotherapy facilities it is not logistically possible to deliver anthracyclines over an extended period. In the facilities in which this review team work, for example, $30 \mathrm{~min}$ of infusion via a $100 \mathrm{ml}$ minibag is the norm for reasons of economy and patient throughput.

There is limited evidence pertaining to the effectiveness of interventions to manage cancer treatmentinduced cardiotoxicity. While two different medical interventions were identified in a systematic review that focused on treatment strategies for cardiotoxicity in childhood cancer (enalapril and phosphocreatine), neither was associated with statistically significant improvement in ejection fraction or mortality.

The largest number of systematic reviews included in this meta-review addressed the prevention of cancer therapy-induced cardiotoxicity. As demonstrated in Fig. 2, few strategies appear to reduce the risk of developing clinical heart failure. These included the avoidance of anthracycline-based chemotherapy (which is routine where cardiac risk before therapy is known), the use of doxorubicin derivatives, a longer anthracycline infusion duration and concomitant administration of the cardioprotective agent dexrazoxane. Of note, all meta-analyses that revealed statistically significant reductions in the rate of clinical heart failure related specifically to the use of anthracyclines. This is not surprising, considering anthracyclines are the focus of the greatest amount of research in this particular field [7]. However, our metareview identified that the Level 1 evidence from metaanalyses focused on the prevention of cardiotoxicity was derived from a relatively small number of trials and in most cases, less than one thousand participants in total. Therefore, despite the fact that the cardiotoxic effects of this particular chemotherapy regimen have been known for a considerable time, there are still gaps in the evidence regarding how to facilitate early detection and management. In particular, the evidence for strategies that protect children with cancer from developing cardiac complications associated with their treatment is lacking [35].

Of note, one previous overview of systematic reviews on the topic of cancer treatment-induced cardiotoxicity has been published [39]. However, this review was smaller in scope than the present review. It focused only on the prevention of cardiotoxicity associated with anthracycline treatment in the paediatric population [39]. Furthermore, only reviews registered by the Cochrane Collaboration were included in van Dalen et al's systematic review [39]. Excluding all other reviews is an effective strategy to ensure only high quality systematic reviews when detailed quality appraisal is not employed as part of the meta-review process [40]. It is possible however that systematic reviews not registered with the Cochrane Collaboration will meet many AMSTAR criteria, indicating that sufficient processes were undertaken to ensure potential sources of bias associated with the systematic review process were avoided. Therefore, including only Cochrane reviews in a meta-review is not the optimal choice when quality appraisal is included as part of the meta-review process.

In regard to the quality of the systematic reviews that reported data on cardiotoxicity, this meta-review identified that: 1) the methodology used in a considerable number of systematic reviews was poor $(\mathrm{n}=11 ; 35 \%$ of the potentially relevant reviews were excluded due to low quality according to the AMSTAR criteria); and 2) half of the systematic reviews not registered with the Cochrane Collaboration were of high quality $(n=9 ; 50 \%$ of reviews that met more than 7 of the AMSTAR criteria were not Cochrane reviews). Based on these findings, it is recommended that future meta-reviews that focus on the prevention, detection and management of cancer treatment-induced toxicities should not include only 
Cochrane reviews, as high-quality systematic reviews that potentially contain unbiased and important recommendations for practice could be overlooked. However, quality appraisal of the systematic reviews would be required to ensure biased conclusions from systematic reviews that have used poor methodology are avoided.

Specific deficiencies in Level 1 evidence for the detection, prevention and management of cancer therapyinduced cardiotoxicity were identified in this meta-review. Only one high quality systematic review of dietary supplementation was identified, which was published in 2004. Recommendations for practice regarding interventions for the detection of cancer treatment-induced cardiotoxicity were not able to be drawn from this meta-review. However, we have identified that an updated systematic review focusing on the detection of cardiotoxicity is required to help inform clinical practice, as the only previous high quality review included evidence up to January 2006. No Level 1 evidence is available to guide clinical decisionmaking regarding the prevention, detection or management of radiation-induced cardiotoxicity. While the role of chest irradiation in inducing cardiotoxicity has been known for some time, studies to date have focused on minimizing the dose of radiation to the heart that are not powered to detect clinical differences in the rate of cardiotoxicity [14]. The small number of primary research studies undertaken to investigate strategies to prevent radiation-induced cardiotoxicity is likely the reason why no systematic reviews were identified in our literature search. While an evidence base about the potential effectiveness of exercise as an intervention to aid prevention of cancer treatment-induced cardiotoxicity is also emerging, similarly, no systematic reviews of the effectiveness of this intervention were identified in our comprehensive search of the literature. Based on the positive results observed in animal studies, it is likely that human clinical trials of exercise for the prevention of cardiotoxicity associated with cancer treatment will be reported in the future [41].

\section{Limitations}

It should be noted that only English language reviews were included in our meta-review. However, we considered this to be acceptable because sensitivity testing regarding information published in languages other than English has shown that English language reviews represent a robust view of the available evidence base in health areas [35]. A considerable strength of this metareview is that we were able to reduce the risk of bias from our conclusions regarding the prevention, detection and management of cancer-treatment induced cardiotoxicity by including only systematic reviews that had considered the quality of included studies in making decisions about the validity of the evidence, as well as the suitability of the included trials for meta-analyses. No attempts were made to combine data from multiple systematic reviews, due to the substantial degree of heterogeneity between the populations, interventions and outcomes investigated. As is the case for all metareviews, it should be noted that evidence from recent studies that were not included in the systematic reviews was not able to be considered in our review. For this reason, the majority of evidence regarding the detection, prevention and management of cancer treatment-induced cardiotoxicity included in this meta-review is from studies conducted at least 5 years ago. Another important point to note is that absolute risk of cardiotoxicity was not reported in meta-analyses due to heterogeneity between individual studies.

\section{Conclusion}

This meta-review has highlighted the paucity of high level evidence to guide clinical practice decision-making regarding the detection and management of cancer treatment associated cardiotoxicity. There is a greater amount of evidence available to guide practice in regard to the prevention of this adverse effect of cancer treatment. It is important to note, however, that the metaanalyses that revealed statistically significant reductions in clinical cardiotoxicity only applied to anthracycline based chemotherapeutic regimens. No high-level evidence is available to guide clinical decision-making regarding the prevention, detection or management of radiation-induced cardiotoxicity.

\section{Additional files}

Below is the link to the electronic supplementary material.

\section{Additional file 1: MEDLINE search strategy. \\ Additional file 2: AMSTAR score of potentially relevant systematic reviews.}

\begin{abstract}
Abbreviations
CoQ10: Coenzyme Q10; HER-2: Anti-human epidermal growth factor receptor 2; cTnT: Cardiac troponin T; ANP: Atrial natriuretic peptide; NT-BNP: N-

terminal nrain natriuretic peptide; ACR: Anthacyclines; LVEF: Left ventricular ejection fraction; HF: Heart failure; 95 \% Cl: 95 \% Confidence interval; RR: Relative risk; OR: Odds ratio; HR: Hazard ratio.
\end{abstract}

\section{Competing interests}

The authors declare that they have no competing interest.

\section{Authors' contributions}

AC: designed the review, acquired data, conducted analysis, interpreted data, drafted the manuscript, approved version to be published and is

accountable for all aspects of the work. AM: conducted analysis, interpreted data, critically revised the manuscript for important intellectual content,

approved version to be published and is accountable for all aspects of the work. PL: acquired data, conducted analysis, critically revised the manuscript for important intellectual content, approved version to be published and is accountable for all aspects of the work. RC: designed the review, conducted analysis, interpreted data, critically revised the manuscript for important intellectual content, approved version to be published and is accountable for all aspects of the work. All authors read and approved the final manuscript. 


\section{Acknowledgements}

This review was funded by a Seeding Grant from the Faculty of Health Sciences, Flinders University and an IHBI MCR grant from the Queensland University of Technology.

\section{Author details \\ ${ }^{1}$ School of Nursing, Institute of Health and Biomedical Innovation, School of Nursing, Institute of Health and Biomedical Innovation, Charles Hospital and School of Nursing, Midwifery and Paramedicine, Park, Adelaide 5001, South Australia. \\ Received: 17 September 2014 Accepted: 29 April 2015 Published online: 07 May 2015} Queensland University Technology, Kelvin Grove Campus, Kelvin Grove, QLD 4059, Australia. ${ }^{2}$ Division of Cancer Services, Princess Alexandra Hospital and Queensland University Technology, Kelvin Grove Campus, Kelvin Grove, QLD 4059, Australia. ${ }^{3}$ Nursing Research \& Practice Development Unit The Prince Australian Catholic University, Brisbane, QLD, Australia. ${ }^{4}$ School of Nursing and Midwifery, Flinders University, 5042 GPO Box 2100, Sturt Road, Bedford

\section{References}

1. Stratigos A, Forsea A, Van Der Leest R, et al. Euromelanoma: a dermatologyled European campaign against nonmelanoma skin cancer and cutaneous melanoma. Past, present and future. Br J Dermatol. 2012;167:99-104.

2. Richards M. The national awareness and early diagnosis initiative in England: assembling the evidence. Br J Cancer. 2009;101:S1-4.

3. Fleissig A, Jenkins V, Catt S, Fallowfield L. Multidisciplinary teams in cancer care: are they effective in the UK? Lancet Oncol. 2006;7:935-43.

4. Rachet $B$, Maringe $C$, Nur $U$, et al. Population-based cancer survival trends in England and Wales up to 2007: an assessment of the NHS cancer plan for England. Lancet Oncol. 2009;10:351-69.

5. De Angelis R, Sant M, Coleman MP, et al. Cancer survival in Europe 19992007 by country and age: results of EUROCARE-5 - a population-based study. Lancet Oncol. 2014;15:23-34.

6. Urruticoechea A, Alemany R, Balart J, Villanueva A, Vinals F, Capella G. Recent advances in cancer therapy: an overview. Curr Pharm Des. 2010;16:3-10.

7. Senkus E, Jassem J. Cardiovascular effects of systemic cancer treatment. Cancer Treat Rev. 2011;37:300-11.

8. Curigliano G, Cardinale D, Suter T, et al. Cardiovascular toxicity induced by chemotherapy, targeted agents and radiotherapy: ESMO clinical practice guidelines. Ann Oncol. 2012;23:vii155-vii66.

9. Higgins JPT, Green S, eds. Cochrane handbook for systematic reviews of interventions Version 5.1.0 [Updated March 2011]. The Cochrane Collaboration. 2011. Available from www.cochrane-handbook.org

10. Chu TF, Rupnick MA, Kerkela R, et al. Cardiotoxicity associated with tyrosine kinase inhibitor sunitinib. Lancet. 2007;370:2011-9.

11. Hutson TE, Figlin RA, Kuhn JG, Motzer RJ. Targeted therapies for metastatic renal cell carcinoma: an overview of toxicity and dosing strategies. Oncologist. 2008;13:1084-96.

12. Cardinale D, Colombo A, Lamantia G, et al. Anthracycline-induced cardiomyopathy. J Am Coll Cardiol. 2010;55:213-20.

13. Wells QS, Lenihan DJ. Reversibility of left ventricular dysfunction resulting from chemotherapy: can this be expected? Prog Cardiovasc Dis. 2010;53:140-8.

14. Chargari C, Kirov KM, Bollet MA, et al. Cardiac toxicity in breast cancer patients: from a fractional point of view to a global assessment. Cancer Treat Rev. 2011;37:321-30.

15. Lipshultz SE, Franco VI, Miller TL, Colan SD, Sallan SE. Cardiovascular disease in adult survivors of childhood cancer. Annu Rev Med. 2015;66:161-76.

16. Shea BJ, Grimshaw JM, Wells GA, et al. Development of AMSTAR: a measurement tool to assess the methodological quality of systematic reviews. BMC Med Res Methodol. 2007;7:10.

17. Toma M, McAlister FA, Bialy L, Adams D, Vandermeer B, Armstrong PW. Transition from meeting abstract to full-length journal article for randomized controlled trials. J Am Med Assoc. 2006;295:1281-7.

18. Moher D. Epidemiology and reporting characteristics of systematic reviews. PLoS Med. 2007:4:e78.

19. Collaboration TC. Cochrane handbook for systematic reviews of interventions version 5.1. 2011. Available from www.cochrane-handbook.org

20. Shea BJ, Hamel C, Wells GA, et al. AMSTAR is a reliable and valid measurement tool to assess the methodological quality of systematic reviews. J Clin Epidemiol. 2009;62:1013-20.
21. Qin Y-Y, Li H, Guo X-J, et al. Adjuvant chemotherapy, with or without taxanes, in early or operable breast cancer: a meta-analysis of 19 randomized trials with 30,698 patients. PLoS One. 2011;6:e26946.

22. Ferguson T, Wilcken N, Vagg R, Ghersi D, Nowak AK. Taxanes for adjuvant treatment of early breast cancer. Cochrane Database Syst Rev. 2007, Issue 4 Art No. CD004421.

23. Viani GA, Afonso SL, Stefano EJ, De Fendi LI, Soares FV. Adjuvant trastuzumab in the treatment of her-2-positive early breast cancer: a metaanalysis of published randomized trials. BMC Cancer. 2007;7:153.

24. Valachis A, Nearchou A, Polyzos NP, Lind P. Cardiac toxicity in breast cancer patients treated with dual HER2 blockade. Int J Cancer. 2013;133:2245-52.

25. Lemos Duarte I, da Silveira Nogueira Lima JP, Passos Lima CS, Deeke Sasse A. Dose-dense chemotherapy versus conventional chemotherapy for early breast cancer: a systematic review with meta-analysis. Breast. 2012;21:343-9.

26. Lord S, Ghersi D, Gattellari M, Wortley S, Wilcken N, Simes J. Antitumour antibiotic containing regimens for metastatic breast cancer. Cochrane Database Syst Rev. 2004, Issue 4. Art No. CD003367

27. Shelley M, Harrison C, Coles B, Staffurth J, Wilt TJ, Mason MD. Chemotherapy for hormone-refractory prostate cancer. Cochrane Database Syst Rev. 2006, Issue 4. Art No. CD005247

28. Van Dalen E, Raphaël M, Caron H, Kremer L. Treatment including anthracyclines versus treatment not in-cluding anthracyclines for childhood cancer. Cochrane Database Syst Rev. 2011, Issue 1 Art. No.: CD006647

29. van Dalen EC, Caron HN, Dickinson HO, Kremer LCM. Cardioprotective interventions for cancer patients receiving anthracyclines. Cochrane Database Syst Rev. 2011, Issue 6 Art No. CD003917

30. van Dalen EC, Michiels E, Caron HN, Kremer L. Different anthracycline derivates for reducing cardiotoxicity in cancer patients. Cochrane Database Syst Rev. 2010, Issue 5. Art No. CD005006

31. van Dalen EC, Van der Pal H, Caron HN, Kremer L. Different dosage schedules for reducing cardiotoxicity in cancer patients receiving anthracycline chemotherapy. Cochrane Database Syst Rev. 2009, Issue 4. Art No. CD005008

32. Smith LA, Cornelius VR, Plummer CJ, et al. Cardiotoxicity of anthracycline agents for the treatment of cancer: systematic review and meta-analysis of randomised controlled trials. BMC Cancer. 2010;10:337.

33. Itchaki G, Gafter Gvili A, Lahav M, Vidal L, Raanani P, Shpilberg O, Paul M. Anthracycline containing regimens for treatment of follicular lymphoma in adults. Cochrane Database Syst Rev. 2013, Issue 7. Art. No. CD008909.

34. Roffe L, Schmidt K, Ernst E. Efficacy of coenzyme Q10 for improved tolerability of cancer treatments: a systematic review. J Clin Oncol. 2004;22:4418-24.

35. Bryant J, Picot J, Baxter L, Levitt G, Sullivan I, Clegg A. Clinical and costeffectiveness of cardioprotection against the toxic effects of anthracyclines given to children with cancer: a systematic review. Br J Cancer. 2007;96:226-30.

36. Bryant J, Picot J, Baxter L, Levitt G, Sullivan I, Clegg A. Use of cardiac markers to assess the toxic effects of anthracyclines given to children with cancer: a systematic review. Eur J Cancer. 2007;43:1959-66.

37. Sieswerda E, Kremer L, Caron H, van Dalen E. The use of liposomal anthracycline analogues for childhood malignancies: a systematic review. Eur J Cancer. 2011;47:2000-8.

38. Sieswerda E, van Dalen EC, Postma A, Cheuk D, Caron HN, Kremer L. Medical interventions for treating anthracycline-induced symptomatic and asymptomatic cardiotoxicity during and after treatment for childhood cancer. Cochrane Database Systematic Reviews. 2011; Issue 9: CD008011.

39. van Dalen EC, Caron HN, Kremer L. Prevention of anthracycline-induced cardiotoxicity in children: the evidence. Eur J Cancer. 2007:43:1134-40.

40. Chan R. Two decades of exceptional achievements: does the evidence support nurses to favour Cochrane systematic reviews over other systematic reviews? Int J Nurs Stud. 2012;49:773-4.

41. Scott JM, Khakoo A, Mackey JR, Haykowsky MJ, Douglas PS, Jones LW. Modulation of anthracycline-induced cardiotoxicity by aerobic exercise in breast cancer current evidence and underlying mechanisms. Circulation. 2011;124:642-50. 